\title{
THE USAGE OF BLOCKCHAIN IN DIGITALIZATION: CASE STUDY ON DOCUMENTARY CREDIT
}

\author{
Adriana CIOCA ${ }^{a *}$, Kassam WEHBE ${ }^{b}$, Delia POPESCU ${ }^{c}$, Constanța POPESCU ${ }^{d}$ \\ ${ }^{a, b, c, d}$ Valahia University, Targoviste, Romania
}

DOI: $10.24818 / \mathrm{IMC} / 2020 / 01.16$

\begin{abstract}
Starting with the year 2012, the Blockchain has attracted the attention of many managers in the world thanks to its usefulness. One of these usages is to guaranty the fluidity of traffic information and merchandises. The migration toward Blockchain based digital environment create a source of wealth for the operators by its secure, immutable and shared database. The premises of the Blockchain is affecting the digital world positively by connecting different systems to the same database. The use of Blockchain will streamline and secure the traffic of information in global trade. Our focus in this study is to fluidify the process of the documentary credit by creating a smart documentary credit while using the Ethereum Blockchain. This feature gives the ability to trespass third party interferences by automating processes. These automations are time beneficiary and profitable for exporters and importers which means that the end user will benefit from a fair trade with the best prices thanks to the Blockchain.
\end{abstract}

KEYWORDS: Blockchain, smart credit, globalization, crypto-money.

\section{INTRODUCTION}

The IT world is continuously changing, traditional operators must quickly find a place in the safe digitalization data systems and to take advantage of the Information Technology innovations. Migration to more sophisticated computer systems creates a new environment that will become a source of wealth for operators, especially after the confidence that has been established through blockchain - based transactions (Hawlitschek et al., 2018). Actors thus find their opportunities through the transparency and security that the blockchain offers to their investments; hence the main actor always remains the investor. Trust and security are the key issues for economic development and growth. This is often forgotten nowadays in economic policy, such as the monetary policy. With respect to the international information trade, in the supply chain, the blockchain is utile for its advanced applications, by connecting any process initiated by the actors through a decentralization of the system and its database, (Abaie \& Rastegary, 2017). The Authors aim is to analyze the efficiency, the confidence, security and cost on the end users, by the utilization of blockchain in documentary credits. Our methodology will start from the specific blockchain concept literature, documentary credits, completed by an experimental method through which several experiences will be carried out concerning the credit documentation as to be able to create virtual automation, based on qualitative and quantitative techniques. To illustrate our aim, we will introduce the concepts of blockchain utilization and documentary credit. Then, we will present the existing Blockchain solutions and point out the type of the companies that could be interested in blockchain technology. And finally, we will propose a prototype of a Blockchain solution that deals with the problem of documentary credit.

\footnotetext{
* Corresponding author. E-mail address: adriana.cioka@gmail.com
} 


\section{BLOCKCHAIN SYSTEM}

\subsection{Understanding the Blockchain concept}

Information in the world economic structure was perceived as a key sector. Transaction of secured information between parties was possible nowadays by the adaptation in a globalized manner of the adequate information management system. Blockchain is a technology that collects information independently and safely to a non-secure and unreliable environment such as Internet without the need to resort towards a trusted third party (Livre blanc, 2016). Among others, it makes possible to envisage new modes of transaction and exchange of information in the field of supply chain management. The Blockchain model and its applications is still in their early stages. The Blockchain is structurally accessible, shared and secure thanks to the consensus algorithms, which are computer programs, whereby the different nodes agree on a result: first, each computer verifies an information, then sends it to the others. Once the information is received, each executes the same algorithm to choose the right result. The appearance of blockchain re-introduce computer's system and re-define control standards. All executives should adapt to changes in respect of transactions secured data, trusted third parties and system's topologies. The blockchain is a distributed database that registers every transaction. It is an architecture database that permits an immutable data storage, secure and transparent. It facilitates the creation of a professional international trade on common ground. However, it is a public record of events that happened in a specific point in time that is totally verifiable (like insurance, title of registry, record of ownership, property, transfer of assets) (Nakamoto, 2008) (Swan, 2015).

In respect of the digital currency, Blockchain technology can help in all systems that have many users and a frequent data insertion. The transfer of digital assets was the innovation behind the bitcoin. The blockchain gathered innovations from the peer to peer network to cryptography (private/public keys) and to decentralization into the blockchain system, that could manage much more decentralized applications. Large companies are investing in the blockchain because it strengthens trust between actors, security of the data, network expansion, visibility and traceability. A Blockchain consists of digital containers holding information of all kinds: transactions, contracts, titles of property, work of arts, etc. All these blocks form a decentralized and shared digital database, which stores and transfers data via the Internet, in a transparent, secure, autonomous way and without central control (Swan, 2015).

\subsection{Efficiency of blockchain}

The efficiency of technology process is mainly based on the quality of the end result and the time achieved. The transparency of this technology is based on the fact that each exchange between its users is recorded as "transaction blocks" which are consecutive thus form a "chain", hence the notion of a Blockchain. Figure 1, "The Model of Blockchain section", illustrates an example of Blockchain where each block contains all transactions for a time period of 10 minutes. The so called "delay" represents the delay time that comes from the enable time (it is set to 10 minutes on the Blockchain Bitcoin). Any input to a transaction is necessarily the output of another transaction. This mechanism allows a higher level of safety to be achieved, which makes it possible to obtain a stronger system (Nakamoto, 2008). 


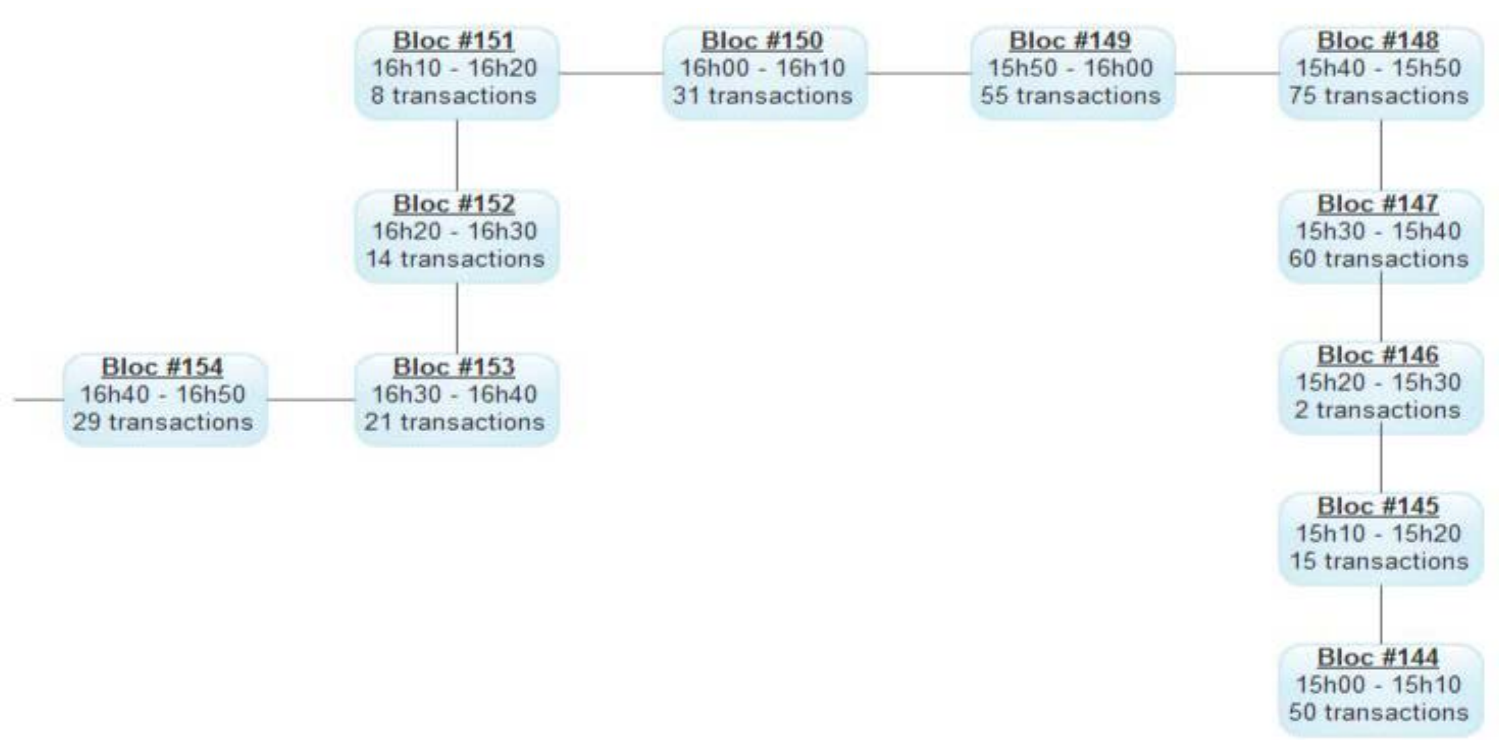

Figure 1. Model of a Blockchain section

Source: The Authors

The principles on which Blockchain is based are as follows:

- Decentralization and disintermediation: no central authority controls the Blockchain; there is no trusted third party.

- Consensus: the fact that a transaction is accepted or rejected is the result of a distributed consensus and not a centralized institution (different forms of consensus exist).

- Immutability: cannot be edited or deleted the entries.

- Shared trust and transparency: sharing of data, operations and consensus as loaded initially.

- Securing: two mechanisms guarantee the structural safety of information recorded within a

- A cryptographic process and the decentralized specific architecture, which enhance security of the data and process efficiency throughout automating tasks (Zhegu \& Olleros, 2016).

\subsection{Blockchain Smart port}

In comparison to the direct traditional documents transfer or by using the intermediary transfer, the PCS (Port Community Systems) and CCS (Cargo Community Systems) are centralized systems with fast time possibility for download. Smart port systems gather, analyze and share information, from and to, as much as systems needed to save space, time, and money in order to reduce ecological footprint. Smart port is the IoT "Internet of Things" oriented where all objects are inter-connected and synchronized. The challenge may be the challenge of multimodal data collection and compatibility of the system. Both smart port and the interface should be flexible with the ability to adapt for changes and developments and aligned in order to synchronize operations as to help inroad inputs data collection for better logistic corridor fluidity and time management and this alignment. However, in Smart Port System, the target is to unify all data retrieved from the supply chain actors, port actors, ports partner and from the port inland captors, to:

- Summarize for a better decision making

- Store useful information for future data analysis

- Be watchful for unexpected event

As an example, traffic information must be retrieved at a moment in time for analyzing road traffic flow management. The particular data collection offers adequate and clear information on upstream, (inland waterways) and downstream (to land threw logistic corridors), which helps Port authorities to improve road/rail traffic monitoring, vessel movements, energy efficiency, access control, supply 
automation, automatic metering, pollution minimizing, maintenance processes, people's location, operations optimizing, cargo tracking, and most important, information exchange (Ilin et al., 2019).

\subsection{The Crypto-currency Bitcoin \& Ethereum network}

\subsubsection{The Crypto-currency}

Crypto currency is a virtual currency inside a Blockchain allowing transfers of values from an account to another account, instantly. It is a form of digital money based on mathematics, where encryption techniques are used to regulate the production of currency units and check the transfer of funds. In addition, crypto-currencies function is independently of a central bank. The appearance of cryptocurrencies, or virtual currencies, is a logical sequel to the movement of freedom that inspired the internet. The Blockchain Bitcoin is a free, open technology that operates as a peer-to-peer network, without central authority and which allows the exchange of units. The creation of Bitcoins is supported by the network and its design. No one owns or controls the Blockchain Bitcoin, and everyone can join. The operation of the Blockchain Bitcoin follows four steps: (1) two persons agree on a transaction; (2) thanks to Blockchain, the transaction is encrypted and validated by consensus (proof of work / mining); (3) it is then added and lock the last block of the Blockchain; (4) finally, the Blockchain is replicated in all nodes of the network (Shirai, 2020).

\subsubsection{The Ethereum network}

Ethereum is a decentralized network distributed across a platform that allows to all users to create their own public, secure, unfalsifiable database, and thus guard against corruption, fraud or data deletion. Ethereum Blockchain can also be used to program "smart contracts", the code of which is a replica of the execution of a standard contract. "Smart contracts" are stand-alone programs that eliminate the risk of fault by one counterparty and enhance quality between all parties. Ethereum is a global computer, which anyone can program and use as it wishes. Its computational power comes from miners who collectively perform the necessary operations (verification, addition of data, execution of smart contracts). The technology behind Ethereum is the same as it is used by the Bitcoin: computers participate in a single public and shared database (the Blockchain), e.g. by sending a message to an application, this message passes through the application server before it reaches to its destination. The Ethereum method allows a decentralized application to be used, the message is sent to an independent network of computers all over the world, and each computer performs a small part of the work (Bogner et al., 2016) (Figure 2: The Ethereum method using a decentralized application).

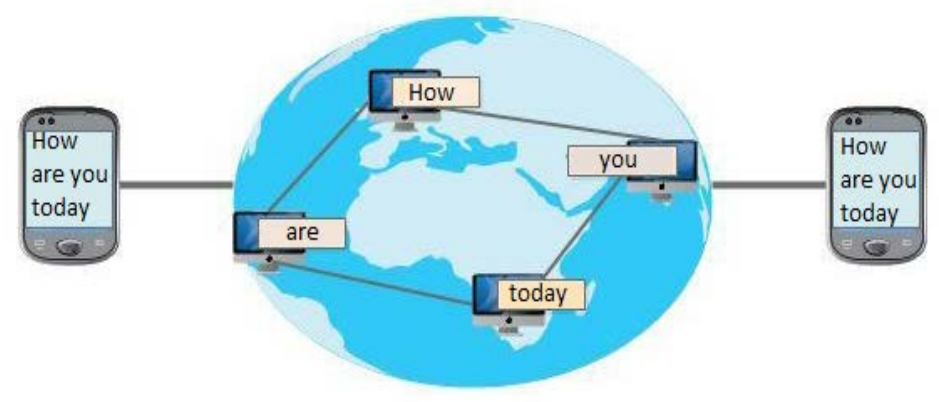

Figure 2. The Ethereum method using a decentralized application Source: The Authors

Bitcoin and Ethereum are open-source platforms that share common features such as the use of Blockchain, a decentralized consensus mechanism based on a proof of calculation, and miners who support the network. However, they have differences in block size, speed at which a block can be mined, programming languages, and so on. 
Block size:

- Bitcoin: the maximum size of a block is limited to avoid spam / Ethereum: it is not limited by a maximum block size.

Block Time (the speed at which a block can be mined):

- Bitcoin: A block is mined every 10 minutes / Ethereum is faster than Bitcoin: the block is mined every $10-19$ seconds.

Programming language:

- There is no programming language on the Bitcoin Blockchain / Ethereum has its own programming language "Solidity": this means that everything can be calculated in this network. (Siriwardena, 2017).

\subsection{The usage of Blockchain \& Smart contracts}

Since the introduction of Bitcoin technology in 2008 and its implementation in 2009, all attention has been focused on the currency and not on the underlying Blockchain technology. There are opportunities to use Blockchain in other areas than money, such as transportation, health, real estate, e-governance, finance, fair trade, etc (Underwood, 2016). All the assets involving an expensive or fallible trusted third party can a priori be disrupted by Blockchain technology. It is possible to categorize Blockchain uses into three types: asset transfers, Blockchain as registry and smart contracts (Underwood, 2016). The Blockchain was initially used in the single area of finance and for the transfer of assets via its use in crypto-currencies, and for monetary transactions. But its application could go beyond its present primary utility. It can be used for transferring value: real estate transaction, crowdfunding (participatory financing), online payments, etc. The Blockchain can be used as a storage register to deposit data to be guaranteed as a proof of existence, date of creation and right of ownership, such as: patents, medical data, etc. (Buffet, 2016). According to Blockchain France, the "smart contract" refers to "stand-alone programs which, once started, automatically execute conditions defined in prior and function's that are like any conditional statement " if-then " type (if this condition is satisfied, then such a consequence executes). Smart contracts reduce audit, execution, arbitration and fraud costs. They can overcome moral hazard problems. The advantage of setting up smart contracts in a Blockchain is to ensure that the terms of the contract cannot be changed (Simon, 2020). The American cryptographer Nick Szabo gave the example of a rented car, whose smart contract could automatically restore control of the car's operation to the lessor if the tenant fails to make payments on time (Nasdaq, 2020). "Slock.it" is one of the Blockchain applications that uses smart contracts. It is defined as the network for the future in a coordinated economy whereas all actors collaborate, with the slogan: "rent, sell or share any object - without intermediary. Its objective is to connect the Blockchain Ethereum with real physical objects. The application is based on a smart lock, called a slock. As for an example: apartment rental: when connecting the lock of the door to the Blockchain, it is possible to link this lock to a "smart contract" rental. When someone wants to use the apartment, it pays the rental price to the contract that is tied to the lock on the Blockchain (Tual, 2020). Once the payment has been made, the door opens automatically for the person who paid the rent for the duration corresponding to the payment. In practice, everything is managed by "smart contracts" in a transparent way, the lease is made by means of an application and payment is made in crypto currency. Typically, smart contracts are at the heart of Ethereum Blockchain applications (Tual, 2020). However, there are projects, such as "Rootstock", which aim to allow the implementation of smart contracts on the Blockchain Bitcoin Nevertheless, there are many benefits of the Blockchain: Profits are mainly ethical and financial. From an ethical point of view, the transparency and the disintermediation of the Blockchain make it possible to gain confidence. Users have more power over simplified processes and higher quality information. The processes are engraved, and the data rendered inalterable. Financially, the architecture of a Blockchain is much less expensive than a centralized technology since it requires fewer machines, little support and the data 
are unalterable. In some situations, the Blockchain can significantly reduce transaction times (especially comparing with banks) (RSK, 2020).

\section{STUDY ON THE BLOCKCHAIN PROCESS WITH THE SCOPE TO FACILITATE THE INTERNATIONAL TRADE}

\subsection{Documentation credit model process with Bank involvement}

The starting point in this particular process represents the documentation credit which is a technique of payment in the context of a financial operation to secure and favor international trade transactions. This commitment brings together a chain of actors linked by a contract. The latter are in general suppliers, buyers and their banks. They must communicate to each other tangible documents as supporting documents for the payment of the documentary credit in accordance with the contractual conditions. However, the security of international transactions by a commercial contract has a fairly high cost (which can reach 2 to $3 \%$ of the total amount of the transaction) ("documentary credit," n.d.). During a trade exchange between an exporter and an importer (buyer/ Seller, customer / supplier), the use of a documentary credit is very important to ensure the smooth operation of the transaction, especially in the case of international trade. In order to do this, it is necessary to respect the various steps mentioned in Figure 3, "The process of a credit model documentation". Throughout this figure it can be observed the main banking route creating the guarantees for the buyer and seller.

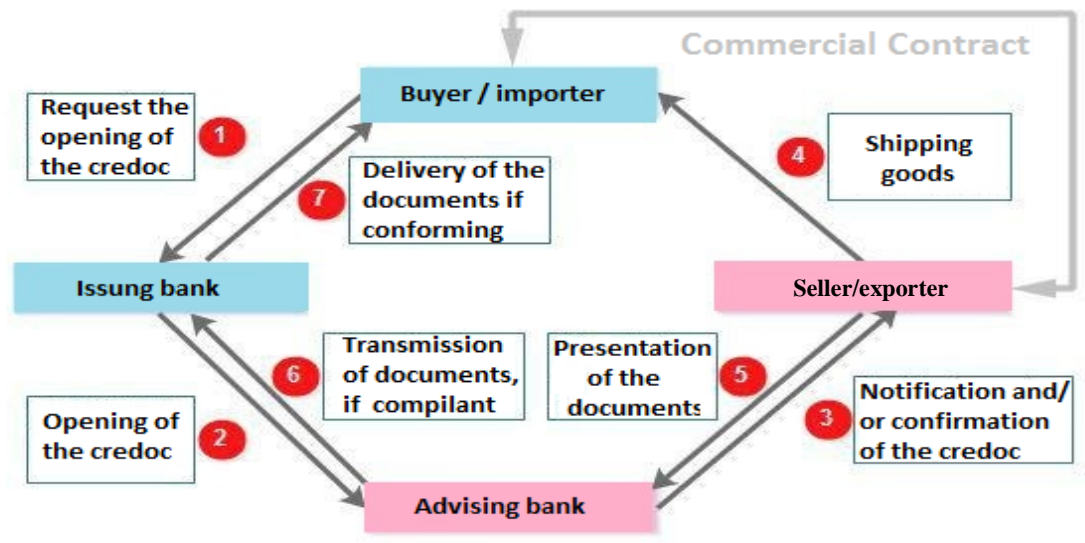

Figure 3. The process of credit model documentation

Source: The Authors

Description of the model:

- The buyer asks his bank (issuing bank) to open a documentary credit with the bank of the seller (notifying bank), on behalf of the latter.

- The issuing bank sends all the documents to the notifying bank, specifying all the conditions for opening the documentary credit: price, quality, quantity, delivery time, etc.

- The notifying bank shall notify this credit opening and shall send the confirmation if the buyer requests.

- The seller checks whether the conditions are in accordance with the commercial contract. If everything is correct, it ships the goods.

- At the same time, the seller collects all the documents required in the credit and delivers them to his bank (notifying bank).

- If the documents comply with the terms of the credit opening, they will be sent to the issuing bank. 
- The documents are transmitted to the buyer, who then takes possession of the goods. The commitment to pay banks is based solely on the strict compliance of the documents.

The principle consists in bringing together the actors of the chain on the same system or "distributed register", the importer (client) and his bank (so-called issuer) on the one hand, and the exporter (seller) and his bank (Called notifier) on the other hand, in order to carry out and validate in real time all the steps necessary for the proper functioning of the documentary credit. Any other actor participating in the exchange must have access to this system, such as the carrier or the port. The Blockchain will ensure consistency and certify the signatures and documents exchanged. In this case, each actor will only have to consult, modify or deposit documents in the Blockchain, which avoids the paperwork and the traditional tedious exchanges. Figure 4, "Documentation credit relationship with the blockchain" shows the progress of the documentary credit regarding the integration of Blockchain.

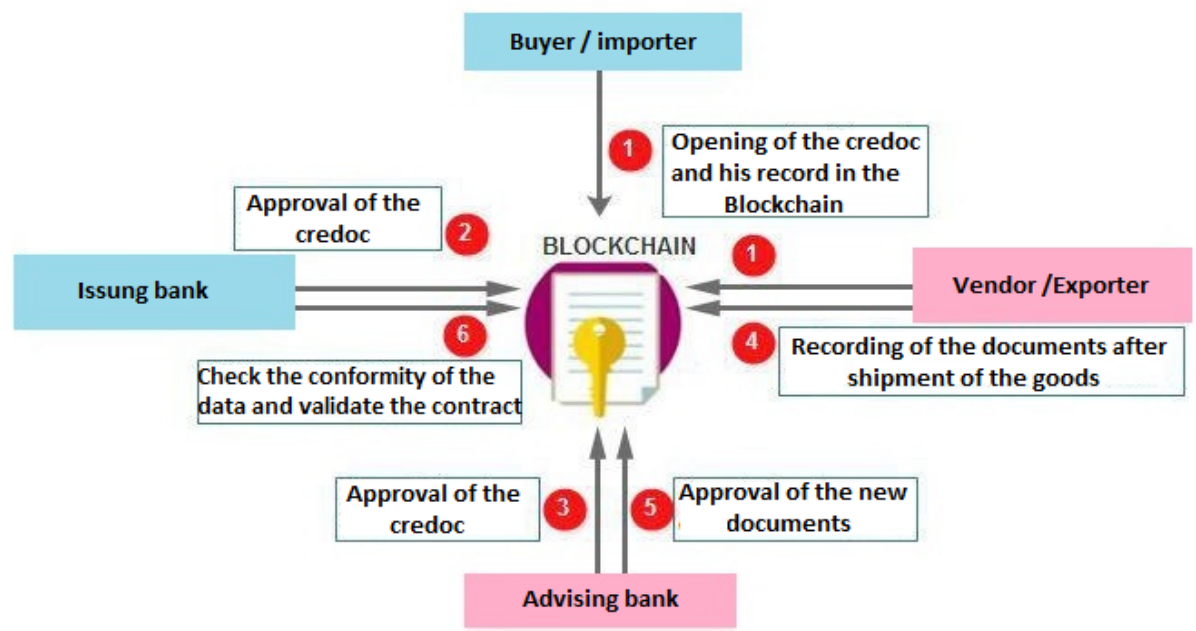

Figure 4. Documentation credit relationship with the blockchain

Source: The Authors

1. After negotiation of the payment by the documentary credit and the agreement on the conditions, the buyer asks the seller to confirm the terms of the order (mode of transport, insurance, etc.) Buyer issue a proforma invoice. The seller enters the invoice directly into the Blockchain, it can contain the following fields: date of invoice, description of the goods, amount, VAT, expiry date, mode of transport, etc.).

2. On the Blockchain, the issuing bank can see that the buyer has validated the invoice. It can then validate the issuance of the documentary credit.

3. The notifying bank may see the validation by the issuing bank of the documentary credit as well as the seller.

4. The seller delivers the goods to the carrier and records the shipping documents in the Blockchain. The carrier in turn delivers to the customer and validates the correct receipt.

5. The notifying bank shall approve the new documents registered by the seller.

6 . The issuing bank checks the conformity of the data, validates the contract and makes the payment to the notifying bank (bank of the seller).

There are also other possibilities not listed, such as customs clearance and customs clearance of goods, or underwriting of currency hedges, etc. However, it is true that this prototype considerably reduces the complexity of the use of documentary credit in terms of time and cost, but it does not reduce the number of intermediaries involved in the exchange operation. To do this, we proposed another prototype that allows us to involve the minimum number of actors, which we will detail in the next section. 


\subsection{Proposed prototype for the documentary credit without banks}

In this context, the principle of the Smart Contracts usage, mentioned above (in the section 2.5) guarantees the security of the transactions, as well as the crypto money to make the payments automatically. In this figure the Authors show the phases needed while having the ports authorities' systems connected to verify that products have been verified to verify automatically without having to present a single paper document.

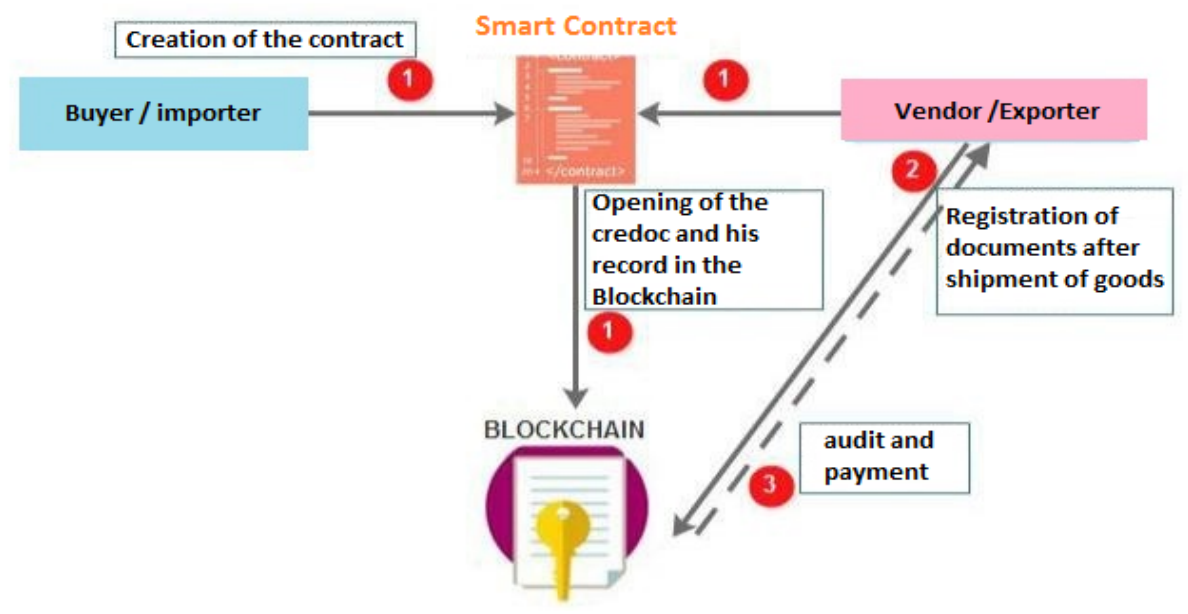

Figure 5. Documentation credit model without Bank involvement Source: The Authors

\section{Process Description:}

1. The buyer and the seller have virtual currency portfolios; they negotiate payment by documentary credit and agree on the conditions. Then, they write a computer program called Smart Contract that capsules the details of their contract. The seller enters the invoice in the Blockchain. This transaction must contain the Smart Contract code.

2. The seller delivers the goods to the carrier and records the shipping documents in the Blockchain. The carrier delivers to the customer and validates the correct receipt.

3. The Smart Contract checks automatically that all conditions of the contract have been met and transfers a virtual currency transfer to the seller.

The prototype has greatly reduced the risk of errors, the number of intermediary interventions, the complexity of processing and verifying the data and documents exchanged. Since the issuing and notifying banks no longer required their involvement, the cost and time required to set up the documentary credit have been optimized. For this prototype, the Authors are using the public Ethereum Blockchain because of:

- effortless programming Smart Contracts.

- possibility of carrying out financial transactions thanks to crypto currency.

The Authors have presented various Blockchain solutions that exist. With respect to the Blockchain solutions adapted to the port systems as well as the companies that are interested in this technology. Finally, the Authors proposed prototypes on the documentary credit that helps for the transportation of goods using the principle of Blockchain. Blockchain technology still an emerging innovation and continues to develop day after day. It has a positive impact on different fields and in this study, we focus on documentary credit simplification. This technology can meet the needs of fluidification, and secured data exchange offered by the Blockchain in order to obtain a safe, fluid, fast and low-cost system. 


\section{THE AUTHORS HAVE DEFINED THE LIMITATIONS OF THE PROTOTYPE BLOCKCHAIN ANALYSIS}

The Blockchain is an emerging architecture which knows many technological limits:

- Blockchain security is problematic at the end of the chain: if the end user loses his private key and transactions are executed without his consent, the data will be unalterable.

- In order for the Blockchain to be secured, it must continually increment new blocks in order to secure the previous blocks. If the sum of the computing powers of the honest users is less than the calculation sum of the users wanting to cheat, then the Blockchain will be corrupted.

- The Blockchain continually records new information without ever deleting it, so we have a problem of exponential storage that is not a priori unreliable in the long term.

- The performance problem (the Bitcoin mining keep on getting harder to solve the problems of the Proof of Work in order to add a block: in some cases, the cost of electricity becomes higher than the profitability to mine) or problems Scalability (Bitcoin can process no more than 7 transactions per second) (Haliplii et al., 2020).

\section{CONCLUSIONS}

Blockchain technology is still under development, it could be in the unavoidable future, from document certification to transactions between individuals or others. It allows data protection, the authenticity of the documents sent as well as the storage of the data as it uses a database which contains the history of all the exchanges that have been made since its creation. However, this exponential growth of the Blockchain could pose problems of storage and synchronization in the future. The Authors presented the Blockchain technology which has considerable advantages, notably in the automation and fluidification of data exchange and security of transfer of assets. It is a database that contains the history of all the exchanges between its users since its creation. This database is secure, transparent and shared by its different users, which allows each user to check the authenticity of the chain. Data digitization facilitates the transmission, sharing of information and improves the environmental efficiency of the port. Most ports are interested in the concept of Smart Ports thanks to its remarkable advantages in terms of energy consumption and the environment. In analyzing the utilization of the blockchain in documentary credit, by introducing the concept of open and decentralize systems to transportation, by connecting all transportation ecosystem into one network and one platform and creating a system of systems that interacts with data, objects and users it reduced time.

\section{REFERENCES}

Bogner, A., Chanson, M., \& Meeuw, A. (2016). A Decentralised Sharing App running a Smart Contract on the Ethereum Blockchain. Proceedings of the 6th International Conference on the Internet of Things - IoT'16.

Buffet G., Comprendre la Blockchain. Anticiper le potentiel de disruption de la blockchain sur les organisations - LLB -. Jan. 2016.

Abaie H. R. \& Rastegary M. The Emergence of Smart Ports and Their Impactful Implications. 24th25th January 2017

Haliplii, R., Guegan, D., \& Frunza, M. (2020). To mine or not to mine? The bitcoin mining paradox. SSRN Electronic Journal. https://doi.org/10.2139/ssrn.358351

Hawlitschek, F., Notheisen, B., \& Teubner, T., (2018). The limits of trust-free systems: A literature review on blockchain technology and trust in the sharing economy. Electronic Commerce Research and Applications, 29, pp.50-63. 
Ilin, I., Jahn, C., Weigell, J., \& Kalyazina, S. (2019). Digital Technology Implementation for Smart City and Smart Port Cooperation. Proceedings of the International Conference on Digital Technologies in Logistics and Infrastructure (ICDTLI 2019).

Livre blanc : Comprendre la blockchain : Anticiper Le potentiel de disruption de la blockchain sur Les organisations. (2016, January). Les livres blancs | La plateforme de référence du livre blanc et de l'ebook. https://www.leslivresblancs.fr/livre/informatique-et- logiciels/blockchain /comprendre-la-blockchain-anticiper-le-potentiel-de-économiste

Revue du commerce international - Crédoc/Crédit documentaire. Revue-du-commerceinternational.info. Retrieved 6 September 2020, from http://www.revue-du-commerceinternational.info/fr/documentation/credoc.

Nakamoto, S. (2008). Bitcoin: A peer-to-peer electronic cash system. Retrieved 12 May 2019, from http://www.cryptovest.co.uk/resources/Bitcoin\%20paper\% 20Original.pdf. Qu'est-ce qu'Ethereum? Blockchainfrance.net. (2020). Retrieved 4 March 2020, from https://blockchainfrance.net/2016/03/04/comprendre-ethereum/.

RSK. Rsk.co. (2020). Retrieved 6 February 2020, from https://www.rsk.co.

Shirai, S., 2020. Money And Central Bank Digital Currency.

Simon Polrot. Qu'est-ce que l'Ethereum? (2016). Retrieved 12 Mars 2020, from https://www.ethereum-france.com/quest-ce-que-lethereum/

Siriwardena, P. (2017). The Mystery Behind Block Time. Medium. Retrieved 2 March 2020, from https://medium.facilelogin.com/the-mystery-behind-block-time-63351e35603a.

Smart Contracts Described by Nick Szabo 20 Years Ago Now Becoming Reality. Nasdaq. (2020). Retrieved 17 July 2020, from https://www.nasdaq.com/articles/smart-contracts-described-bynick-szabo-20-years-ago-now-becoming-reality-2016-04-26.

Tual, S. (2020). IoT \& Blockchain Technology for Renting, Selling, Sharing - Tech Talks Central. Tech Talks Central. Retrieved 16 March 2020, from https://techtalkscentral.com/interview/iotblockchain-technology-renting-selling-sharing/.

Swan, M., (2015). Blockchain. Sebastopol, CA: O'Reilly Media, Inc.

Underwood, S. (2016). Blockchain beyond bitcoin. Communications Of The ACM, 59(11), 15-17. https://doi.org/10.1145/2994581

Zhegu, M. \& Olleros, F. (2016). Research Handbook On Digital Transformations. Northampton, MA: Edward Elgar Pub. 